\title{
Subgap Current through the Strongly Correlated Quantum Dot Hybridized with the Normal and Superconducting Leads
}

\author{
T. Domański And A. Donabidowicz \\ Institute of Physics, M. Curie-Skłodowska University \\ pl. M. Curie-Skłodowskiej 1, 20-031 Lublin, Poland
}

\begin{abstract}
We investigate charge tunneling through the strongly correlated quantum dot placed between the metallic and superconducting leads. For small voltages $|V| \leq \Delta / e$ applied across the junction the current is transmitted by the anomalous mechanism of the Andreev reflections. Such transport is sensitive to the strong Coulomb interactions which can be responsible for the charging effect and, at low temperatures, for appearance of the Kondo resonance. We analyze their signatures in the differential Andreev conductance.
\end{abstract}

PACS numbers: 73.23.-b, 74.20.Fg, 74.45.+c

\section{Introduction}

The recent development of nanotechnology enables a controllable manipulation of a hybridization between electrons on the small size islands (where the quantum effects play a remarkable role) and the external reservoirs of itinerant charge carriers. In this paper we investigate such single "quantum dot" (QD) located between the metallic and superconducting electrodes. This situation can be explored under non-equilibrium conditions applying the source-drain voltage which triggers the charge tunneling. At low bias the subgap current is transmitted solely through the Andreev reflections [1] and these in turn are sensitive to the QD spectrum as well as the proximity effect $[2-5]$. We shall discuss the effective spectrum of the strongly correlated quantum dot. Furthermore, we consider the optimal conditions for observation of the zero-bias feature induced in the differential conductance by the Kondo resonance when spin of the quantum dot is screened by electrons of the metallic lead.

\section{Microscopic model and the methodology}

To describe the strongly correlated quantum dot placed between the normal $(\mathrm{N})$ and superconducting $(\mathrm{S})$ electrodes we use the Anderson model

$$
\begin{aligned}
\hat{H} & =\hat{H}_{\mathrm{N}}+\hat{H}_{\mathrm{S}}+\sum_{\sigma} \epsilon_{d} \hat{d}_{\sigma}^{\dagger} \hat{d}_{\sigma}+U \hat{n}_{d \uparrow} \hat{n}_{d \downarrow} \\
& +\sum_{\boldsymbol{k}, \sigma} \sum_{\beta=\mathrm{N}, \mathrm{S}}\left(V_{\boldsymbol{k} \beta} \hat{d}_{\sigma}^{\dagger} \hat{c}_{\boldsymbol{k} \sigma \beta}+\text { h.c. }\right),
\end{aligned}
$$

where $d_{\sigma}\left(d_{\sigma}^{\dagger}\right)$ are the annihilation (creation) operators for electron of spin $\sigma$ and the energy level $\varepsilon_{d}$. The on-dot repulsion between opposite spin electrons is denoted by $U$ and the last term is a hybridization of the
QD and external leads. As usual, we describe the metallic lead by $\hat{H}_{\mathrm{N}}=\sum_{\boldsymbol{k}, \sigma} \xi_{\boldsymbol{k} \mathrm{N}} \hat{c}_{\boldsymbol{k} \sigma \mathrm{N}}^{\dagger} \hat{c}_{\boldsymbol{k} \sigma \mathrm{N}}$ while the superconducting one is taken in the BCS form

$$
\begin{aligned}
\hat{H}_{\mathrm{S}} & =\sum_{\boldsymbol{k}, \sigma} \xi_{\boldsymbol{k} S} \hat{c}_{\boldsymbol{k} \sigma \mathrm{S}}^{\dagger} \hat{c}_{\boldsymbol{k} \sigma \mathrm{S}} \\
& -\sum_{\boldsymbol{k}} \Delta\left(\hat{c}_{\boldsymbol{k} \uparrow S}^{\dagger} \hat{c}_{-\boldsymbol{k} \downarrow S}^{\dagger}+\hat{c}_{-\boldsymbol{k} \downarrow S} \hat{c}_{\boldsymbol{k} \uparrow S}\right)
\end{aligned}
$$

with $\xi_{\boldsymbol{k} \beta}=\varepsilon_{\boldsymbol{k} \beta}-\mu_{\beta}$. External voltage $V$ detunes the chemical potentials by $\mu_{\mathrm{N}}=\mu_{\mathrm{S}}+e V$.

Effective spectrum of the QD can be determined considering the retarded Green function $\boldsymbol{G}(\tau)=$ $\left\langle\left\langle\hat{\Psi}(\tau) ; \hat{\Psi}^{\dagger}\right\rangle\right\rangle$ defined in the Nambu representation $\hat{\Psi}^{\dagger}=$ $\left(\hat{d}_{\uparrow}^{\dagger}, \hat{d}_{\downarrow}\right), \hat{\Psi}=\left(\hat{\Psi}^{\dagger}\right)^{\dagger}$. For simplicity we focus on the situation when the energy level $\varepsilon_{d}$ is located deep inside the gap of superconductor, i.e. $|\omega| \ll \Delta$. The Dyson equation simplifies then to $[6,7]$ :

$$
\begin{aligned}
& \boldsymbol{G}(\omega)^{-1}=\left(\begin{array}{cc}
\omega-\varepsilon_{d} & 0 \\
0 & \omega+\varepsilon_{d}
\end{array}\right) \\
& \quad-\left(\begin{array}{cc}
0 & \frac{1}{2} \Gamma_{\mathrm{S}} \\
\frac{1}{2} \Gamma_{\mathrm{S}} & 0
\end{array}\right)-\left(\begin{array}{cc}
\Sigma_{\mathrm{N}}(\omega) & 0 \\
0 & \Sigma_{\mathrm{N}}^{*}(-\omega)
\end{array}\right),
\end{aligned}
$$

where $\Gamma_{\beta}=2 \pi \sum_{\boldsymbol{k}}\left|V_{\boldsymbol{k} \beta}\right|^{2} \delta\left(\omega-\xi_{\boldsymbol{k} \beta}\right)$. The second term on the right hand side of (2) comes from hybridization of the QD with the superconducting lead and is responsible for the proximity effect, i.e. for a non-vanishing expectation value $\left\langle\hat{d}_{\downarrow} \hat{d}_{\uparrow}\right\rangle$. The last term in (2) represents a contribution to the self-energy from the normal lead. In the extreme limit $\Delta \rightarrow \infty$ it has been shown analytically [6] that such matrix has a diagonal structure for arbitrary $U$.

Neglecting the correlations one can obtain the exact solution $\Sigma_{\mathrm{N}}(\omega)=-\mathrm{i} \Gamma_{\mathrm{N}} / 2$. Effective spectrum of the QD consists then of two Lorentzian peaks at 
$E_{d}= \pm \sqrt{\varepsilon_{d}^{2}+\Delta_{d}^{2}}\left(\right.$ where $\left.\Delta_{d}=\Gamma_{\mathrm{S}} / 2\right)$ and their spectral weights are given by the BCS coefficients $\frac{1}{2}\left[1 \pm \varepsilon_{d} / E_{d}\right]$. Effect of the Coulomb interactions has been studied by a number of approximate techniques (see e.g. Ref. [8] for a recent review). In the present work we impose the ansatz based on the equation of motion study [9]:

$$
\omega-\varepsilon_{d}-\Sigma_{\mathrm{N}}(\omega)=\frac{\left.\left[\omega-\varepsilon_{d}-\Sigma_{0}(\omega)\right]\left\{\omega-\varepsilon_{d}-\left[U+\Sigma_{0}(\omega)+\Sigma_{3}(\omega)\right]\right\}+U \Sigma_{1}(\omega)\right]}{\omega-\varepsilon_{d}-\left[\Sigma_{0}(\omega)+\Sigma_{3}(\omega)+U\left(1-n_{d, \sigma}\right)\right]},
$$

where

$$
\begin{aligned}
& \Sigma_{\nu}(\omega)=\sum_{\boldsymbol{k}}\left|V_{\boldsymbol{k N}}\right|^{2}\left(\frac{1}{\omega-\xi_{\boldsymbol{k N}}}+\frac{1}{\omega-U-2 \varepsilon_{d}+\xi_{\boldsymbol{k N}}}\right) \\
& {[f(\omega, T)]^{\frac{3-\nu}{2}}}
\end{aligned}
$$

and $n_{d, \sigma}=\left\langle\hat{d}_{\sigma}^{\dagger} \hat{d}_{\sigma}\right\rangle, f(\omega, T)=[1+\exp (\omega / T)]^{-1}$. This approach (3) has a virtue to capture the following main aspects of the Coulomb interactions: (a) the charging effect and (b) appearance of the Kondo resonance at low temperatures.

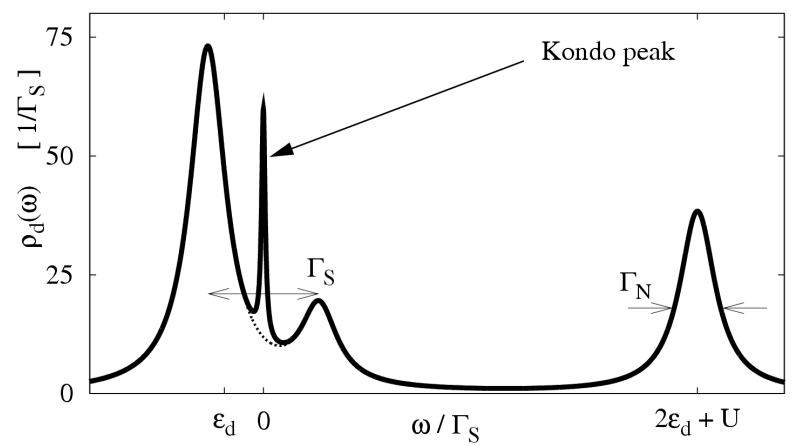

Fig. 1. Spectral function $\rho_{d}(\omega)$ for the strongly correlated quantum dot in the Kondo regime for $\Gamma_{\mathrm{N}}=\Gamma_{\mathrm{S}} / 2$, $\varepsilon_{d}=-\Gamma_{\mathrm{S}} / 2, U=5 \Gamma_{\mathrm{S}}, T=10^{-4} \Gamma_{\mathrm{S}}$ and $\Delta \rightarrow \infty$ assuming the equilibrium conditions $(V=0)$.

Figure 1 illustrates the spectrum of QD in equilibrium situation $(V=0)$ for the Kondo regime assuming the energy level located slightly below the Fermi energy $\varepsilon_{d}=-\Gamma_{\mathrm{S}} / 2$ and $U=5 \Gamma_{\mathrm{S}}$. We can notice that effective spectrum is formed in a vicinity of two atomic levels $\varepsilon_{d}$ and $2 \varepsilon_{d}+U$ corresponding to the singly and doubly occupied QD configurations. These states have roughly the Lorentzian shapes and their broadening is given by a coupling to the normal lead $\Gamma_{\mathrm{N}}$ (we consider here the limit of large $\Delta$ therefore the superconducting lead does not contribute any broadening [10]). On top of this atomic-like spectrum we observe appearance of the low energy superstructure due to: (i) the particlehole splitting induced by the proximity effect $[6,7,11]$, and (ii) the narrow Kondo resonance at $\omega=0$ caused by the antiferromagnetic coupling between QD and electrons of the metallic lead [2-5]. The first effect is thus inherited from the superconducting lead while the latter one comes solely from the on-dot correlations supported by the metallic lead electrons. Both effects compete with one another in much the same way as magnetism competes with isotropic singlet superconductivity in the solid state physics. This important issue is discussed by us in more detail elsewhere [12].

Applying external bias $V$ between the electrodes induces the charge current

$$
I(V)=-e \frac{\mathrm{d}}{\mathrm{d} t}\left\langle\sum_{\boldsymbol{k}, \sigma} \hat{c}_{\boldsymbol{k} \sigma \mathrm{N}}^{\dagger} \hat{c}_{\boldsymbol{k} \sigma \mathrm{N}}\right\rangle .
$$

In the present case we focus on small voltages $|e V| \ll \Delta$, when the charge is transmitted only by the mechanism of the Andreev reflections [1]. Such anomalous current can be expressed by the following formula $[4,10]$ :

$$
\begin{gathered}
I_{\mathrm{A}}(V)=\frac{2 e}{h} \int \mathrm{d} \omega \Gamma_{\mathrm{N}}^{2}\left|\boldsymbol{G}_{12}\left(\omega+\mathrm{i} 0^{+}\right)\right|^{2} \\
\times[f(\omega+e V, T)-f(\omega-e V, T)] .
\end{gathered}
$$

At low temperatures the differential conductance $G_{\mathrm{A}}(V)=\frac{\mathrm{d}}{\mathrm{d} V} I_{\mathrm{A}}(V)$ is proportional to the off-diagonal part of the matrix Green function - it is hence useful for studying the interplay between the particle-hole splitting and the Kondo effect.
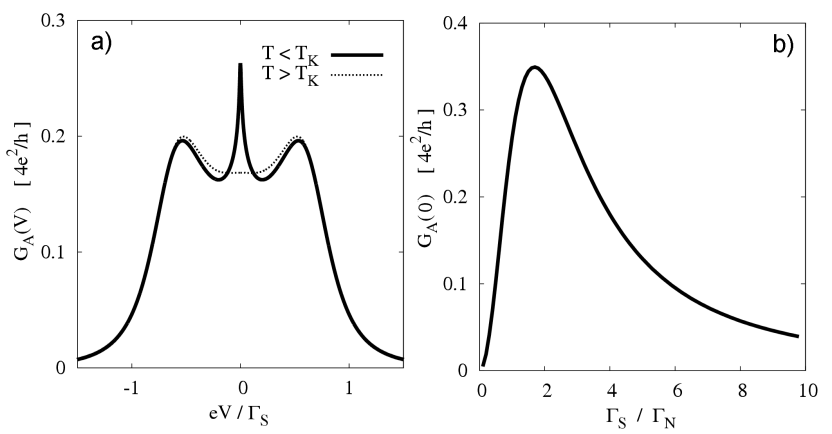

Fig. 2. (a) Andreev conductance $G_{\mathrm{A}}(V)$ for the same set of parameters as used in Fig. 1. (b) The zero-bias Andreev conductance versus the coupling ratio $\Gamma_{\mathrm{S}} / \Gamma_{\mathrm{N}}$ computed at low temperature $T=10^{-4} \Gamma_{\mathrm{S}}$.

In the left hand side part of Fig. 2 we show the Andreev conductance obtained above the Kondo temperature $T_{\mathrm{K}}$ (the thin dashed curve corresponds to $T=100 T_{\mathrm{K}}$ ) and in the Kondo regime (the thick solid line). We notice that in both cases there appear two flat maxima at $\mathrm{eV}= \pm \Delta_{d}$ because of the particle-hole splitting. Furthermore, the low temperature Kondo resonance affects the Andreev current by increasing the zero-voltage conductance [11]. 
To determine optimal conditions for the zero-bias enhancement caused by the Kondo effect we have explored numerically $G_{\mathrm{A}}(0)$ versus the ratio $\Gamma_{\mathrm{S}} / \Gamma_{\mathrm{N}}$. The results shown in Fig. $2 \mathrm{~b}$ indicate that such situation occurs for $\Gamma_{\mathrm{S}} \sim \Gamma_{\mathrm{N}}$. We hope that our finding would stimulate some further experimental search either confirming or invalidating the presence of such zero-bias feature in N-QD-S junctions. So far there have been reported the indications for the zero-bias anomaly in S-QD-S junctions but there this effect probably originates from the Josephson current [13].

\section{Conclusions}

We have considered the correlated quantum dot located between the metallic and superconducting electrodes. The on-dot Coulomb interactions are responsible for the charging effect and, at sufficiently low temperatures, lead to appearance of the Kondo resonance. The latter effect originates from screening of the QD spin by mobile electrons from the metallic electrode. The low voltage (subgap) Andreev conductance is found to show the unique zero-bias feature related to the Kondo effect. The magnitude of zero-bias enhancement is optimal for $\Gamma_{\mathrm{S}} \sim \Gamma_{\mathrm{N}}$. Upon increasing $\Gamma_{\mathrm{S}}$ the particle-hole splitting takes over and the QD is driven to the mixed valence regime suppressing the zero-bias anomaly.

\section{Acknowledgments}

This work is partly supported by the Ministry of Science and Education under the grants NN202187833 and NN202373333.

\section{References}

[1] G.E. Blonder, M. Tinkham, T.M. Klapwijk, Phys. Rev. B 25, 4515 (1982).

[2] A. Levy Yeyati, J.C. Cuevas, A. López-Dávalos, A. Martín-Rodero, Phys. Rev. B 55, R6137 (1997).

[3] R. Fazio, R. Raimondi, Phys. Rev. Lett. 80, 2913 (1998); Phys. Rev. Lett. 82, 4950 (1998); P. Schwab, R. Raimondi, Phys. Rev. B 59, 1637 (1999).

[4] Q.-F. Sun, J. Wang, T.-H. Lin, Phys. Rev. B 59, 3831 (1999).

[5] H.-K. Zhao, J. Wang, Eur. Phys. J. B 44, 93 (2005).

[6] A. Oguri, Y. Tanaka, A.C. Hewson, J. Phys. Soc. Jpn. 73, 2494 (2004).

[7] Y. Tanaka, N. Kawakami, A. Oguri, J. Phys. Soc. Jpn. 76, 074701 (2007).

[8] J. Splettstoesser, M. Governale, J. König, F. Taddei, R. Fazio, Phys. Rev. B 75, 235302 (2007).

[9] R. Świrkowicz, J. Barnaś, M. Wilczyński, Phys. Rev. B 68, 195318 (2003); H. Haug, A.-P. Jauho, Quantum Kinetics in Transport and Optics of Semiconductors, Springer Verlag, Berlin 1996.

[10] M. Krawiec, K.I. Wysokiński, Supercond. Sci. Technol. 17, 103 (2004).

[11] T. Domański, A. Donabidowicz, K.I. Wysokiński, Phys. Rev. B 76, 104514 (2007).

[12] T. Domański, A. Donabidowicz, Phys. Rev. B 78, 073105 (2008).

[13] A. Eichler, M. Weiss, S. Oberholzer, C. Schönenberger, A. Levy Yeyati, J.C. Cuevas, A. Martín-Rodero, Phys. Rev. Lett. 99, 126602 (2007); C. Buizert, A. Oiwa, K. Shibata, K. Hirakawa, S. Tarucha, Phys. Rev. Lett. 99, 136806 (2007). 